\title{
Músculo Elevador de la Escápula: Irrigación e Inervación
}

\author{
Levator Scapulae Muscle: Blood Supply and Innervation \\ Felipe Mardones Valdivieso \& Alberto Rodríguez Torres
}

MARDONES, V. F. \& RODRIGUEZ, T. A. Músculo elevador de la escápula: Irrigación e inervación. Int. J. Morphol., 24(3):363-368, 2006.

RESUMEN: La utilización de colgajos musculares como herramienta reconstructiva requiere un acabado conocimiento anatómico de la irrigación e inervación del tejido muscular donante. Las aplicaciones quirúrgicas del músculo elevador de la escápula han contribuido a profundizar este conocimiento, pese al cual, persisten interrogantes respecto a estas variables morfológicas.

Se trabajó con 11 cadáveres (ocho masculinos y tres femeninos), con una edad promedio de 70 años. A siete de ellos se les había hecho previamente una repleción vascular. En nueve preparados se disecaron ambos lados de las regiones cervical y escapular y en dos, sólo un lado. Se estudiaron distintas variables de la vascularización arterial e inervación.

Identificamos cuatro fuentes de irrigación: arterias vertebral (AV), cervicales ascendente (CA) y transversa (CT) y dorsal de la escápula (DE). El riego sanguíneo en su mayoría ocurrió por la combinación entre ellas, siendo los pedículos dominantes principalmente ramas de CT y DE. La irrigación efectiva del músculo fue diferente para cada arteria: $100 \%$ para $\mathrm{AV}, 80 \%$ para $\mathrm{CA}, 85 \%$ para $\mathrm{CT}$ y un $55 \%$ para DE. Se determinó la ubicación topográfica de los pedículos vasculares: AV irriga los orígenes tendinosos del músculo, CA irriga principalmente el segmento superior, CT y DE irrigan los segmentos intermedio e inferior. El patrón de irrigación muscular en su mayoría correspondió al tipo V, según la clasificación de Mathes y Nahai.

La inervación provino principalmente desde ramos del tercer y cuarto nervio espinal cervical (C3 y C4) (64.7\%), aunque el quinto nervio (C5) también participó por intermedio del nervio dorsal de la escápula. Los puntos motores para C3 y C4 se ubicaron en el segmento superior del músculo, mientras que para C5 se ubicó en el segmento inferior.

La irrigación generosa y proveniente desde distintas fuentes arteriales junto a una inervación segmentaria, confirman las bondades de este músculo al considerarlo como colgajo muscular.

PALABRAS CLAVE: Irrigación; Inervación; Músculo elevador de la escápula.

\section{INTRODUCCIÓN}

El empleo de colgajos musculares es una herramienta importante y difundida en el campo de la cirugía reconstructiva actual. Dentro de las variables morfológicas que son fundamentales para el éxito de esta intervención están la irrigación e inervación del tejido muscular donante.

Con el advenimiento de nuevas técnicas y aplicaciones quirúrgicas, ha sido necesario profundizar en el conocimiento de estas variables mencionadas. Si bien la experiencia publicada en la utilización del músculo elevador de la escápula como elemento reconstructivo es escasa, se le han atribuido importantes aplicaciones (Marks \& Cummings, 1985; Goodman \& Donald, 1990; Angrigiani et al., 2003; Romero \&
Gerber, 2003). No obstante lo anterior, el conocimiento actual sobre estos aspectos es aún insuficiente, desincentivando en cierta forma su uso como alternativa reconstructiva. Por ejemplo, existen interrogantes respecto a temas como cuáles son las distintas fuentes arteriales que irrigan el músculo, cuál es su distribución y la ubicación topográfica de los pedículos vasculares. Asimismo, se encuentra escasa información sobre la distribución topográfica de los ramos nerviosos al músculo.

Dada la importancia que tiene tanto la irrigación como la inervación del tejido muscular para la viabilidad de este elemento reconstructivo, nos propusimos realizar un estudio anatómico al respecto. 


\section{MATERIAL Y MÉTODO}

Este trabajo se llevó a cabo en las dependencias de Anatomía Normal de la Universidad de los Andes, Chile. Se utilizaron once cadáveres, ocho de sexo masculino y tres femeninos, todos de raza caucasoide. La edad promedio fue de 70 años (rangos entre 43 a 96 años). Los preparados se encontraban fijados y conservados mediante solución conservadora empleada en dicha Universidad. Además, a siete se les había hecho una repleción arterial con una mezcla de látex natural y colorante (tinta china). Se procedió a una disección por planos, que abarcó las regiones anterior, posterior y lateral del cuello, junto con la región escapular. En nueve de los casos, se disecaron ambas regiones cervicales y escapulares, y dos en sólo un lado, dando un total de veinte casos para estudio. Una vez completada la disección, se analizaron lo siguientes aspectos:

\section{Vascularización arterial:}

Frecuencia de arterias que participaron en la irrigación del tejido muscular. Asimismo, se analizaron las combinaciones posibles entre ellas.

Dominancia de cada arteria o de combinaciones entre éstas en la irrigación del músculo. Para esto fueron considerados el grosor y número de rama(s) del(los) pedículo(s) vascular(es) que llegó(aron) al tejido muscular.

Longitud del pedículo vascular principal, tomando la distancia desde el sitio de entrada de la arteria dominante al músculo hasta su arteria originaria.

Territorios de irrigación de cada arteria nutricia, considerando la ubicación de los pedículos vasculares.

Tipo (patrón) de irrigación del músculo, basándose en la clasificación de Mathes y Nahai.

\section{Inervación:}

Ramos eferentes que llegaban al músculo. Punto motor para cada fascículo muscular.

Para realizar las mediciones de longitud de los elementos vasculares, se utilizó una estructura flexible (hilo) el cual era, posteriormente, colocado sobre un instrumento métrico.

Con el fin de ubicar topográficamente los sitios de llegada de los pedículos vasculares y puntos motores, designamos como segmento superior a la porción del músculo que va desde su origen vertebral craneal y profundo al músculo esternocleidomastoídeo. La porción del músculo entre el primer segmento y el borde anterolateral del músculo trapecio se denominó segmento intermedio. Por último, el segmento inferior correspondió a aquel situado entre la porción precedente hasta su sitio de inserción escapular.

Dada la disposición escalonada y en sentido cráneocaudal de los fascículos musculares, designamos como F1 al primer fascículo, es decir a aquel que tenía su origen más craneal con respecto a los otros. Luego a los fascículos caudales a F1 y en orden descendente, se les designó como F2, F3, F4 o F5, según el número total de fascículos que presentó cada músculo.

Finalmente, se tomaron fotografías de los preparados con el fin de ilustrar los hallazgos más relevantes.

\section{RESULTADOS}

Vascularización arterial: El aporte arterial al músculo provino de 4 fuentes: arterias cervicales transversa (CT) y ascendente (CA), dorsal de la escápula (DE) y vertebral.

La arteria vertebral se encontró en todos los preparados y dio origen a pequeñas ramas que irrigaron los orígenes tendinosos de los fascículos musculares. Asimismo, ramas originadas de la arteria vertebral se anastomosaron con ramas de la arteria cervical ascendente. La arteria cervical ascendente se encontró en 19 preparados, de los cuales irrigó efectivamente el músculo en 16, encontrándose una relación de 84.21\%. En el caso de la arteria cervical transversa también se encontró en 19 casos, irrigando el músculo en 17 preparados, con una relación de $89.70 \%$. Finalmente, identificamos a la arteria dorsal de la escápula en 15 preparados, de los cuales irrigó a 11, con una relación de 73.33\%. (Figs. 1 y 2). El riego sanguíneo muscular ocurrió de manera independiente o mediante la combinación entre las arterias mencionadas: $\mathrm{CA}+\mathrm{CT}$ en 8 casos $(40 \%)$, $\mathrm{CA}+\mathrm{CT}+\mathrm{DE}$ en 6 casos $(30 \%), \mathrm{CT}+\mathrm{DE}$ y $\mathrm{CA}+\mathrm{DE}$ con 2 casos $(10 \%)$ cada uno y, finalmente, CT y DE con 1 caso $(5 \%)$ cada uno. Se determinó la existencia del (los) pedículo (s) que irriga (ron) de forma dominante: CT en 9 casos (45\%), DE en 7 casos $(35 \%), \mathrm{CT}+\mathrm{DE}$ en 3 casos $(15 \%)$ y $\mathrm{CA}+\mathrm{CT}+\mathrm{DE}$ en 1 caso (5\%). En la Tabla I, se muestran los territorios del músculo que eran irrigados por cada arteria en particular.

Basándonos en la clasificación de Mathes \& Nahai, en 16 casos $(80 \%)$ el patrón de irrigación del músculo correspondió al tipo V, en el resto (20\%) fue compatible con el tipo II.

Finalmente, se midió la longitud del pedículo dominante, lo cual dio como promedio $2.82 \mathrm{~cm}$. (D.E \pm 0.96 ). 


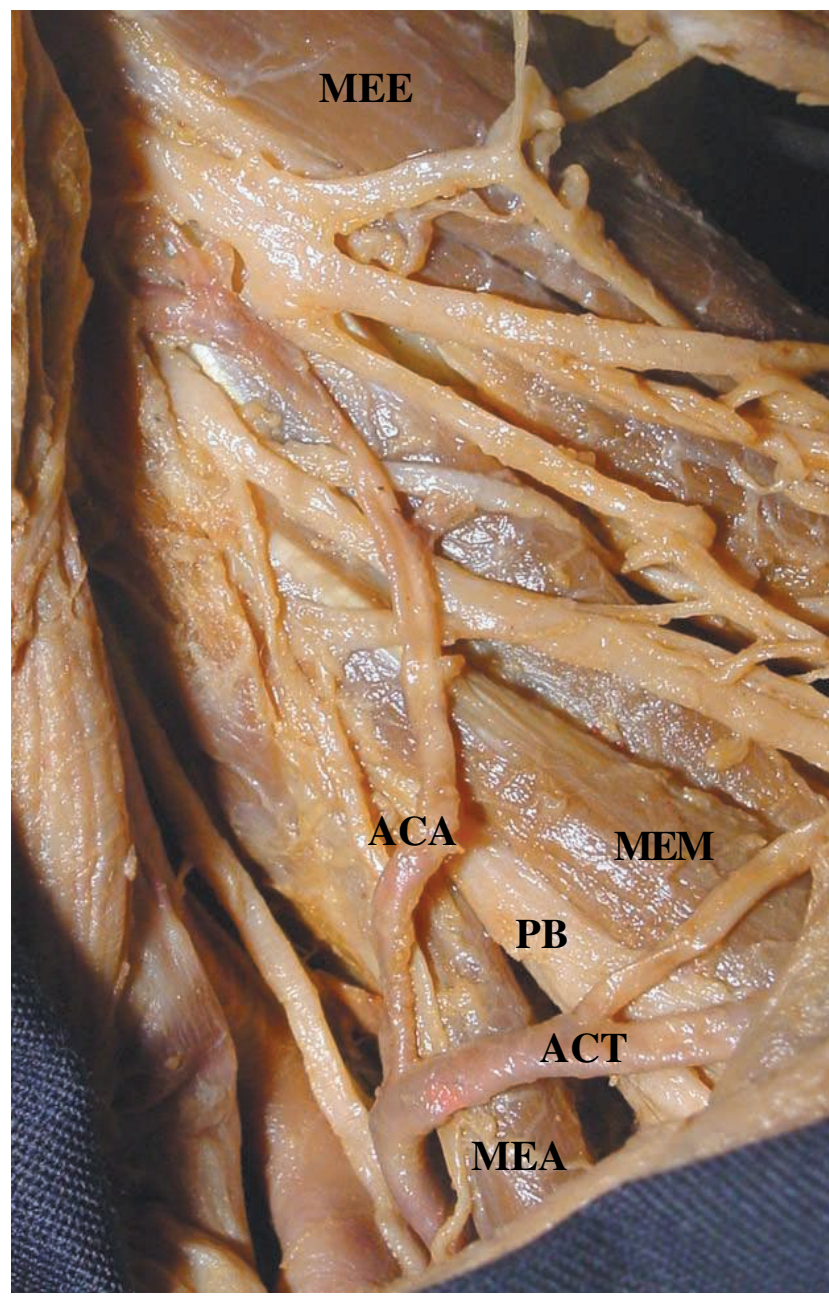

Fig. 1. Visión lateral izquierda del cuello. Irrigación del músculo elevador de la escápula (MEE). Arterias cervical ascendente (ACA) y cervical transversa (ACT), músculos escaleno anterior (MEA) y medio (MEM), plexo braquial (PB).

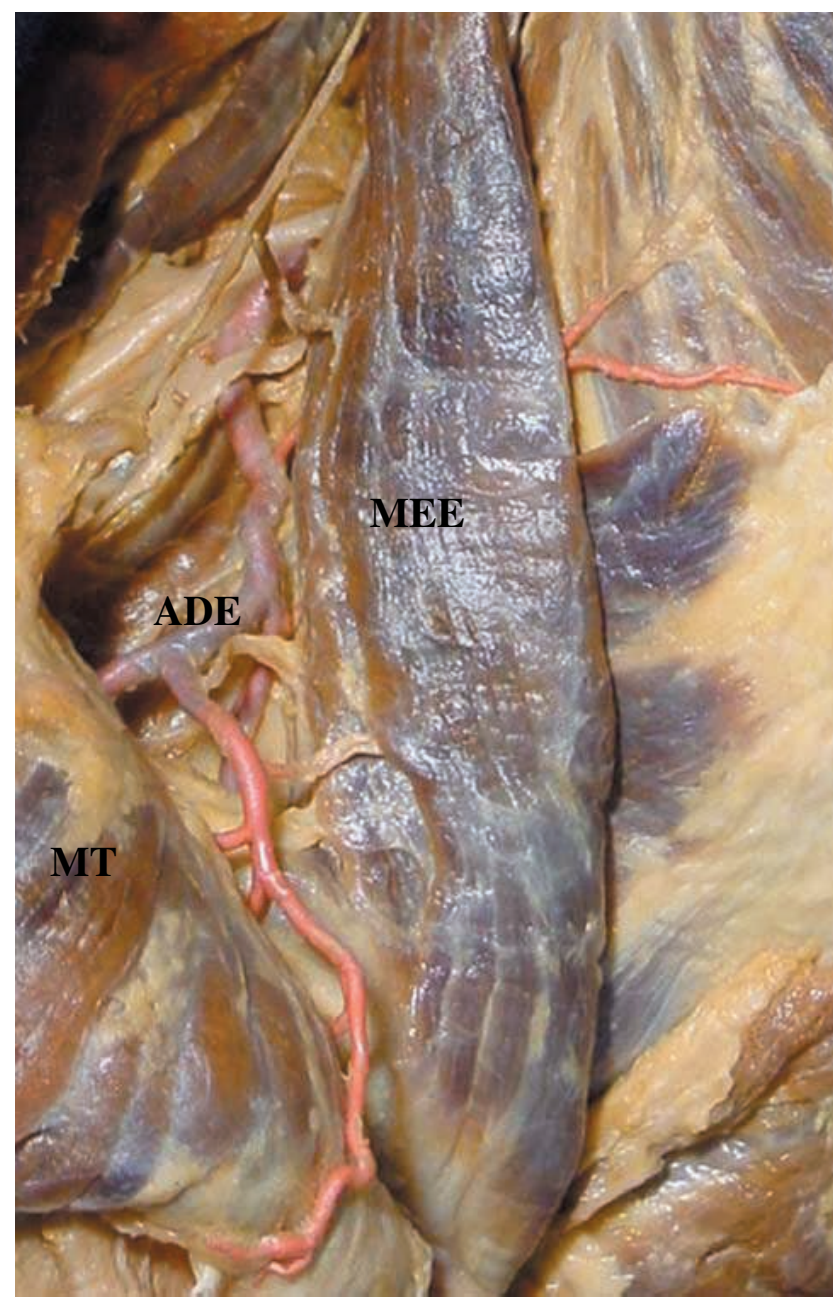

Fig. 2. Visión lateral izquierda del dorso. Se aprecian pedículos vasculares de la arteria dorsal de la escápula (ADE) llegando al músculo elevador de la escápula (MEE). Músculo trapecio (MT.)

Tabla I. Territorios de irrigación del músculo elevador de la escápula.

\begin{tabular}{clcc}
\hline Arteria & Ubicación (segmento-cara) & $\begin{array}{c}\text { Número de } \\
\text { casos }\end{array}$ & $\begin{array}{c}\text { Frecuencia } \\
(\mathbf{\%})\end{array}$ \\
\hline Cervical ascendente & superior - posterolateral & 1 & 6,25 \\
& superior - anterolateral & 12 & 75,0 \\
& superior - medial & 2 & 12,5 \\
& intermedio - borde anterior & 1 & 6,25 \\
Cervical transversa & intermedio - anterior & 2 & 11,76 \\
& intermedio - anterolateral & 2 & 11,76 \\
& intermedio - medial & 1 & 5,88 \\
& inferior - anterolateral & 10 & 58,22 \\
& inferior - posterior & 2 & 11,76 \\
Dorsal de la escápula & intermedio - borde anterior & 1 & 6,25 \\
& intermedio - posterolateral & 2 & 12,5 \\
& inferior - inferior & 2 & 12,5 \\
& inferior - posterolateral & 5 & 31,25 \\
& inferior - posterior & 1 & 6,25 \\
& inferior - medial & 5 & 31,25 \\
\hline
\end{tabular}


Inervación: El músculo elevador de la escápula recibe ramos eferentes profundos del plexo cervical. La inervación motora provino de los nervios espinales cervicales tercero y cuarto(C3 y C4, respectivamente), o bien, la suma de los dos anteriores más el quinto nervio espinal cervical (C5); este último, por intermedio del nervio dorsal de la escápula. De acuerdo a la indemnidad de los elementos nerviosos estudiados, se obtuvo que 11 casos $(64.70 \%)$ fueron inervados por $\mathrm{C} 3+\mathrm{C} 4$ y en $6 \operatorname{casos}(35.29 \%)$ por $\mathrm{C} 3+\mathrm{C} 4$ + C5. (Fig.3)

Se determinó en qué segmento del músculo se ubicaba el punto motor para cada nervio espinal evaluado. Asimismo, fue determinado qué fascículo(s) era(n) inervado(s) por cada uno. Tabla II.

En su trayecto descendente hacia los músculos romboides, el nervio dorsal de la escápula inervó al músculo elevador de la escápula, ya sea perforándolo o dando ramos que se desprendían del tronco nervioso y llegaban hasta el músculo. En nuestros preparados, encontramos que en 2 de los 6 seis casos totales (33.33\%), el nervio perforó al músculo, inervándolo. En los otros 4 casos $(66.67 \%)$, salieron filetes nerviosos, entre 1 a 2, que llegaron a las fibras musculares.

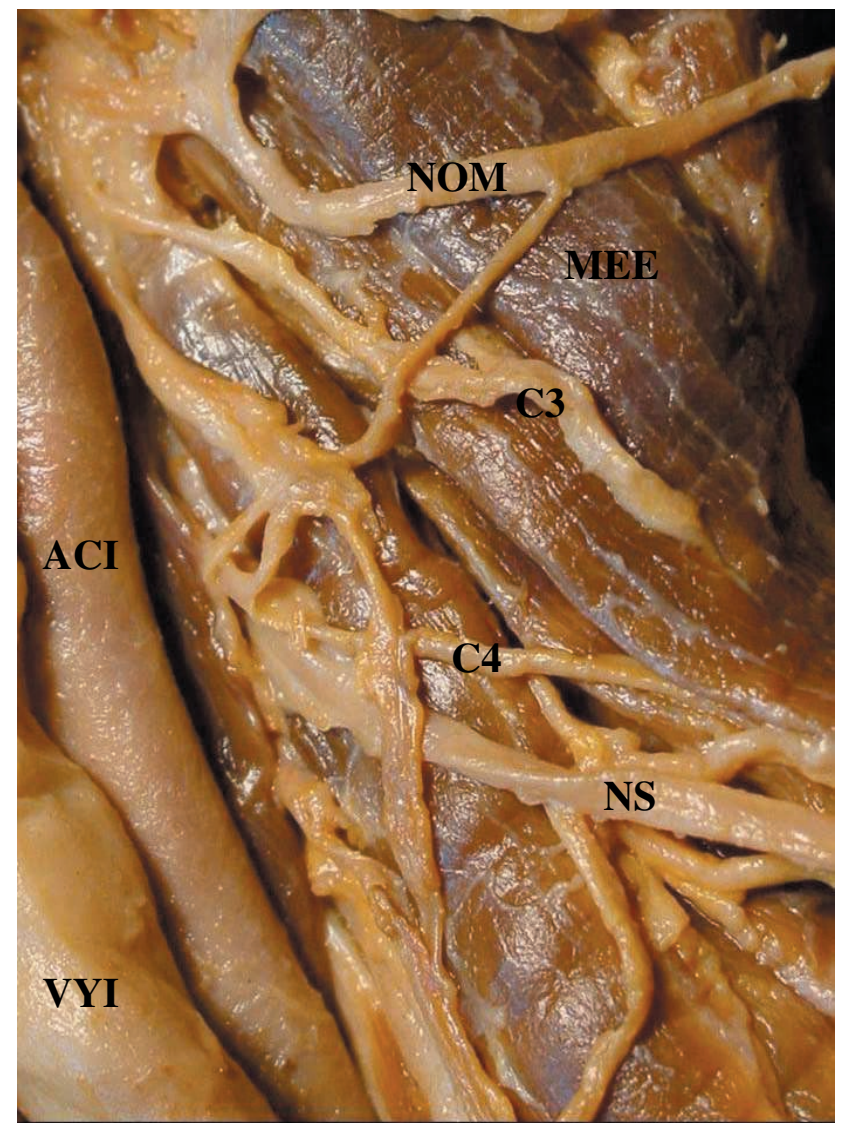

Fig. 3. Visión lateral izquierda del cuello. Se aprecian ramos motores de los nervios espinales cervicales tercero y cuarto (C3 y $\mathrm{C} 4)$ llegando al músculo elevador de la escápula (MEE). Arteria carótida interna (ACI), vena yugular interna (VYI), nervios supraclavicular (NS) y occipital menor (NOM).

Tabla II. Inervación y punto motor de fascículos musculares.

\begin{tabular}{cccc}
\hline Nervio Espinal & Punto motor & Número de casos & Frecuencia $(\%)$ \\
\hline C3 & 3 & 20,00 \\
& Segmento superior - borde anterior & 12 & 80,00 \\
Segmento superior - cara lateral & & \\
Fascíc ulo(s) & F1 & 5 & 41,67 \\
F1 + F2 & 7 & 58,33 \\
& & 12 & 92,30 \\
C4 & 1 & 7,69 \\
& Segmento superior - cara anterolateral & & 15,38 \\
& Segmento intermedio - cara lateral & & 38,47 \\
& Fascíc ulo(s) & 5 & 30,77 \\
F2 & 52 & 15,38 \\
C5 + F3 54 & 4 & 16,67 \\
& F3 + F4 + F5 & 2 & 16,67 \\
& & 1 & 66,66 \\
& Segmento inferior - cara anterior & 1 & 25,0 \\
& Segmento inferior - cara posterior & 4 & 62,5 \\
Segmento inferior - cara medial & Fascíc ulo(s) & 2 & 12,5 \\
\hline
\end{tabular}




\section{DISCUSIÓN}

El conocimiento anatómico de la irrigación de un músculo es un elemento importante a considerar, previo a su elección como colgajo. Asimismo, en las técnicas en que se pretende utilizar el colgajo como sustituto funcional, la inervación del mismo es fundamental. En el caso del músculo elevador de la escápula, existe información respecto al tema, a nuestro parecer insuficiente y a veces contradictoria tanto en los textos anatómicos clásicos como en la literatura especializada (Paturet, 1951; Hollinshead,1969; Romanes, 1987; Latarjet, \& Ruiz-Liard, 1998) destacando los trabajos de Huelke (1958), Smith et al. (1974), Rodríguez et al. (1989) y Ogawa et al. (2004) sobre los pedículos vasculares del músculo y de Frank et al. (1997) y Tubbs et al. (2005), respecto a su inervación.

En nuestro estudio identificamos varias fuentes posibles de irrigación arterial, todas ellas ramas directas o indirectas de la arteria subclavia. Dentro de éstas, la arteria vertebral, participó principalmente en la irrigación de los orígenes tendinosos de los fascículos musculares. Por su parte, la arteria cervical ascendente, en su trayecto ascendente y superficial a los músculos escalenos dio origen a ramas de pequeño calibre que se dirigieron hacia lateral para finalmente llegar a los fascículos musculares superiores, con mayor frecuencia en los segmentos superior e intermedio por las caras anterolateral, medial y posterolateral en el primero y el borde anterior en el segundo. A su vez, la arteria cervical transversa, presentó un trayecto superficial y transverso a la musculatura lateral del cuello. A lo largo de su recorrido, se originaron ramas para los músculos escalenos, luego, dirigiéndose hacia dorsal dio, por lo general, una rama para el músculo trapecio y otra rama muscular descendente para el músculo elevador de la escápula y romboides. En algunos preparados, también llegaron ramas al elevador de la escápula desde la rama trapezoidal. En general, los pedículos vasculares de esta arteria se ubicaron, en el 70\% de los casos, en el segmento inferior del músculo por su cara anterolateral y en el resto, en el segmento intermedio. La arteria dorsal de la escápula presentó un recorrido hacia dorsal y descendente que fue variable, dado que, en algunos casos, compartió la irrigación de los músculos de esta zona con la arteria cervical transversa; en general, esta arteria dio ramas al músculo elevador de la escápula en su segmento inferior, predominando las caras posterolaterales y mediales (profundas) del mismo. Asimismo, dio ramas musculares al segmento intermedio con una frecuencia cercana al $19 \%$.

$\mathrm{Al}$ analizar la irrigación efectiva del músculo, éste recibió ramas musculares, en la mayoría de los casos, a partir de más de una de las arterias mencionadas anteriormente. Comprobamos que en un $40 \%$ de los preparados, la irrigación provino de la combinación entre las arterias cervicales ascendente y transversa. En un $20 \%$ provino de la sumatoria entre la dorsal de la escápula más la cervical ascendente o la cervical transversa. Cabe destacar que se observó que, en un 30\%, la irrigación efec- tiva llegó de la combinación entre las tres. Finalmente, en sólo un 5\% de los casos las arterias dorsal de la escápula o la cervical transversa por sí mismas irrigaron el músculo.

Nuestros resultados mostraron que las arterias dominantes fueron, en su mayoría, las cervical transversa y dorsal de la escápula, ya sea actuando de forma aislada o bien, mediante la combinación entre ambas.

De acuerdo a la clasificación de los distintos patrones de irrigación muscular según Mathes y Nahai, encontramos dos tipos aludibles. El más frecuente correspondió al tipo V, identificándose un pedículo dominante y varios pedículos secundarios, lo que implica una ventaja quirúrgica en relación a la movilización del colgajo, ya que se puede "sacrificar" uno de los sistemas vasculares y aún así mantener la viabilidad del músculo. Por otra parte, en un $20 \%$ constatamos un patrón de tipo II, es decir, la presencia de varios pedículos dominantes (en su mayoría dos) asociados a uno o varios pedículos secundarios. Al contrario del tipo anterior, la lesión de cualquiera de los dos sistemas vasculares compromete la viabilidad del músculo, con lo que se limita el radio de movilización del colgajo a la longitud de los pedículos. En los trabajos y experiencias publicados (Marks \& Cummings; Goodman \& Donald), se menciona la movilización del músculo sacrificando sus pedículos vasculares inferiores, preservando la irrigación aportada por la arteria cervical ascendente. Cabe destacar que la casuística de estos trabajos era escasa y los resultados mostrados no estuvieron exentos de complicaciones (Goodman \& Donald, 1990). Además, en nuestros preparados, la arteria cervical ascendente no irrigó al músculo, en el $10 \%$ de los casos. Todo lo anterior hace sugerir la conveniencia de estudiar la vascularización arterial mediante imágenes previo al abordaje quirúrgico, con el fin de asegurar la viabilidad del colgajo muscular.

Finalmente, el radio de giro del colgajo muscular (considerando la longitud del pedículo dominante) fue corto, con un promedio de $2.82 \mathrm{~cm}$.

Mediante el estudio de la inervación del elevador de la escápula, comprobamos que recibe ramos eferentes profundos nervios espinales cervicales tercero y cuarto, o bien la sumatoria de éstos más el quinto nervio espinal cervical. En nuestro estudio, la mayor parte de los preparados fue inervado por C3 más C4 (64.7\%); en los casos en que se sumó C5, este último llegó al músculo, ya sea perforando la masa muscular o bien dando ramos más pequeños que se desprendieron del tronco nervioso principal. El ramo eferente de C3, en la mayor parte de los preparados, llegó al músculo a nivel del segmento superior y por la cara lateral, aunque también al mismo nivel; pero por su borde anterior. De esta forma, inervó F1 o bien la sumatoria de F1 y F2. C4 por su parte, puede inervar todos los fascículos a excepción de F1. En la mayoría de los casos, su punto motor se encontró en el segmento superior, por la cara anterolateral del fascículo. 
Finalmente, a través del nervio dorsal de la escápula, C5 inervó mayoritariamente a F4 (62.5\%), aunque también lo hizo para F2 más F3 o sólo F5. Llegó a estos fascículos en el segmento inferior del músculo, en la mayoría de los casos, por su cara medial o bien por sus caras anterior o posterior.

Las características morfológicas descritas confirman algu- nas de las ventajas en la utilización del músculo elevador de la escápula como colgajo muscular. Entre éstas, destacamos la presencia de una irrigación generosa proveniente desde varias fuentes posibles, junto con un patrón de irrigación muscular beneficioso para la movilización. Asimismo, la ubicación topográfica de los pedículos vasculares y puntos motores nerviosos relativamente constantes, facilita la labor del cirujano al abordar este músculo.

MARDONES, V. F. \& RODRIGUEZ, T. A. Levator scapulae muscle: Blood supply and innervation. Int. J. Morphol., 24(3):363-368, 2006.

SUMMARY: The use of muscular flaps as a reconstructive tool requires a thorough anatomical knowledge of its blood supply and innervation. Although surgical applications of the levator scapulae muscle have contributed to this matter, there are still questions regarding these morphologic features. In this study, 11 human corpses were used ( 8 males and 3 females), with an average age of 70 years. In 7 of these, an arterial repletion was performed prior to dissection. The latter was done in a step-wise manner in both sides of 9 cervical and scapular regions and in 2 cases, only one side. Several aspects of the arterial blood supply and innervation of the muscle were analyzed.

We identified 4 possible sources for arterial irrigation: Vertebral (V), ascending (AC) and transverse cervical (TC) and dorsal scapular (DE). Overall, the blood supply occurred as a combination of the above, with TC and DE providing the dominant pedicles in most cases. Differences were noted in the effective muscular irrigation between the arteries, wich was $100 \%$ for $\mathrm{V}, 80 \%$ for $\mathrm{AC}, 85 \%$ for TC and 55\% for DE. Regarding the location of the vascular pedicles, $\mathrm{V}$ irrigated the muscle origins, AC irrigated the upper segment, whereas TC and DE irrigated the middle and lower muscle segments. Based on the Mathes and Nahai classification, the majority had a type $\mathrm{V}$ irrigation pattern.

In most cases, the innervation came from the third and fourth spinal cervical nerve (C3 and C4), although the fifth spinal cervical nerve (C5) also participated through the dorsal scapular nerve. The motor points for C3 and C4 were located in the upper segment of the muscle, whereas for C5 it was located in the lower segment. Due to its generous and heterogeneous arterial supply and segmentary innervation, the muscle is a good alternative to consider if it is to be used as a reconstructive flap.

KEY WORDS: Blood supply; Innervation; Levator scapulae muscle.

\section{REFERENCIAS BIBLIOGRÁFICAS}

Angrigiani, C.; Grilli, D.; Karanas, Y. L.; Longaker, M. T. \& Sharma, S. The dorsal scapular island flap: an alternative for head, neck, and chest reconstruction. Plast. Reconstr. Surg.111(1):67-78, 2003.

Frank, D.; Wenk, E.; Stern, J.; Gottlieb, R. \& Moscatello, A. A cadaveric study of the motor nerves to the levator scapulae muscle. Otolaryngol. Head and Neck Surg., 117(6):671-80, 1997.

Goodman, A. \& Donald, P. Use of the Levator Scapulae Muscle Flap in Head and Neck Reconstruction. Arch Otolaryngol Head Neck Surg., 116:1440-4, 1990.

Hollinshead, W. Anatomy for Surgeons. 2. ed. The Back and Limbs). Harper \& Row Publishers, New York, 1969. V. 3.

Huelke, D. A study of the transverse cervical and dorsal scapular arteries. Anat. Rec., 132(3):233-45, 1958.

Latarjet, M. \& Ruiz-Liard, A. Anatomía Humana. 3ª ed. Panamericana, Madrid, 1998.

Marks, N. \& Cummings, Ch. The levator scapulae muscle flap. A new use. J. Laryngology and Otology, 99:471-4, 1985.

Mathes, S. J. \& Nahai, F. Clinical Applications for Muscle and Musculocutaneaus Flaps. The C.V Mosby Company, St. Louis, 1982.

Ogawa, R.; Hyakusoku, H.; Murakami, M. \& Gao, JH. Clinical and basic research on occipito-cervico-dorsal flaps: including a study of the anatomical territories of dorsal trunk vessels. Plast Reconstr Surg. 113(7):1923-33, 2004.
Paturet, G. Traité d'Anatomie Humaine. Osteologie-Artrologie-Myologie. Masson Editeurs, Paris, 1951. V. 1.

Rodríguez, A.; Lippi, P. \& Medina, A. Arteria Cervical Transversa, Estudio Anátomo-Quirúrgico. Anales de Anatomía Normal, 7:50-3, 1989.

Romanes, G. J. Cunningham Tratado de Anatomía. 12. ed. McGraw-Hill, Madrid, 1987.

Romero, J. \& Gerber, C. Levator scapulae and rhomboid transfer for paralysis of trapezius. The Eden-Lange procedure. J. Bone Joint Surg. Br., 85(8):1141-5, 2003.

Smith, R.; Sanders, W. J. \& Stewart, K. C. Blood Supply to the Levator Scapulae Muscle Relative to Carotid Artery Protection. Trans Am Acad Ophthalmol Otolaryngol, 78(3):128-34, 1974.

Tubbs, R. S.; Tyler-Kabara, E. C.; Aikens, A. C.; Martin, J. P.; Weed, L. L.; Salter, EG. \& Oakes, WJ. Surgical anatomy of the dorsal scapular nerve. J. Neurosurg., 102(5):910-1, 2005.

Dirección para correspondencia:

Dr. Felipe Mardones Valdivieso

Unidad de Anatomía Normal, Facultad de Medicina,

Universidad de los Andes.

Avenida San Carlos de Apoquindo 2200

Santiago, Chile.

Email: fmardonesv@yahoo.com

Recibido : 22-03-2006

Aceptado: 12-06-2006 